\title{
MiR-591 functions as tumor suppressor in breast cancer by targeting TCF4 and inhibits Hippo-YAP/TAZ signaling pathway
}

Xin Huang ${ }^{1}$, Fen Tang ${ }^{1}$, Zeping Weng ${ }^{2}$, Mengyao Zhou ${ }^{3}$ and Qing Zhang ${ }^{1 *}$

\begin{abstract}
Background: MicroRNAs have been involved in regulating crucial biological function in some tumors. However, the clinical role and functional effects of miR-591 in breast cancer remain unknown.

Methods: The expression of miR-591 was detected in breast cancer tissues and their paired normal tissues by qRTPCR. Functional assays were performed to confirm the effects of miR-591 on the proliferation and invasion of breast cancer. Bioinformatics analysis, luciferase reporter assays, western blot and in vitro assays were used to confirm that TCF4 was a target gene of miR-591. Western blot analysis was carried out to analyze the relationship between miR-591 expression and YAP1 expression in breast cancer.

Results: We found that miR-591 expression levels were significantly downregulated in breast cancer tissues compared to adjacent normal tumor tissues. Lower miR-591 expression notably related to lymph node metastasis and advanced TNM stage in patients with breast cancer. In vitro, cell proliferation and invasion were inhibited by transfection of miR-591 mimic in breast cancer cells, but were promoted by transfection of miR-591 inhibitor, compared to the controls. In vivo, we also found that miR-591 mimic significantly inhibited cell proliferation ability. Moreover, we identified that TCF4 was a direct target of miR-591 in breast cancer. TCF4 mediated the inhibiting effects of miR-591 on cell proliferation and invasion in breast cancer cells. In additional, we revealed that miR-591 overexpression significantly inhibited the Hippo-YAP/TAZ signaling pathway in breast cells by downregulated YAP1 expression in breast cells.
\end{abstract}

Conclusion: Together, these results indicated that miR-591 is downregulated in breast cancer and could act as a potential target of breast cancer treatment.

Keywords: Breast cancer, MicroRNAs, miR-591, TCF4, YAP1

\section{Background}

Breast cancer is the most common invasive cancer in women worldwide [1]. About 252, 710 new cases are diagnosed and more than 40,000 women are likely to die from this disease [2]. In spite of the mortality rate of patients with breast cancer has been reduced due to the advances in diagnostic methods and therapeutic strategies [3]. However, patients with this disease present tumor metastasis at the late advance stage, leading

\footnotetext{
*Correspondence: jiangzelan600286@163.com

${ }^{1}$ Department of Breast Surgery, The First Affiliated Hospital of Jinan University, 613 West Huangpu Road, Guangzhou 510630, Guangdong, People's Republic of China

Full list of author information is available at the end of the article
}

to poor outcomes and higher mortality $[3,4]$. Thus, to investigate new therapeutic strategies for breast cancer is needed.

MicroRNAs (MiRNAs), a series of small endogenous RNAs, are identified as crucial regulators in breast cancer development and progression [5]. MicroRNAs can regulate downstream gene expression by binding to the $3^{\prime}$-untranslated regions of their target mRNAs [6, 7]. For some microRNAs have been reported to act as regulators in breast cancer progression. MicroRNA-218 inhibits proliferation and invasion in ovarian cancer by targeting Runx2 [8]. MiR-139-5p modulates radiotherapy resistance in breast cancer by repressing multiple gene networks of DNA repair and ROS defense [9]. MiR-17-5p 
suppresses cell proliferation and invasion by targeting ETV1 in triple-negative breast cancer [10]. Moreover, more and more microRNAs were reported to participate in breast cancer biological function.

MiR-591 is identified as tumor suppressor in some tumors. A study showed that upregulation of miR-591 could confer paclitaxel resistance to ovarian cancer [11]. MiR-591 overexpression cellular growth, proliferation, and invasive capability in malignant pleural mesothelioma (MPM) [12]. In the study, we found that miR-591 expression was significantly downregulated in breast cancer tissues. In vitro, we revealed that miR-591 could inhibited cell proliferation and invasion by regulating TCF4 expression. In vivo, we also found that miR-591 mimic significantly inhibited cell proliferation ability. Moreover, we demonstrated that miR-591 overexpression inhibited the Hippo-YAP/TAZ signaling pathway in breast cells by downregulated YAP1 expression. Thus, these results indicated that miR-591 may function as a potential target of breast cancer treatment.

\section{Materials and methods}

\section{Clinical tissue samples and cell lines}

A total of 78 pairs of human breast cancer tissue samples and corresponding adjacent normal tissues were obtained from patients who underwent resection at Department of Breast Surgery, The First Affiliated Hospital of Jinan University between August 2014 and July 2016. All of the patients were female and the median age of the patients was 50 years (range from 28 to 76 years old). The study was approved by the Ethical Committee of The First Affiliated Hospital of Jinan University (the protocol number IACUC-20170602-01) and all of the patients provided written informed consent. Pathological classification and staging were based on the 2007 International Breast Cancer Typing Guidelines.

Four human BC cell lines MCF-7, MDA-MB-231, T-47D, SKBR3 and a human normal breast epithelial cell line MCF-10A were purchased from the Institute of Biochemistry and Cell Biology, Type Culture Collection of the Chinese Academy of Sciences (Shanghai, China). Cells were cultured in Dulbecco's modified Eagle's medium (DMEM) supplemented with 10\% fetal bovine serum (FBS, Gibco; Thermo Fisher Scientific, Inc., Waltham, MA, USA) in a humidified $5 \% \mathrm{CO}_{2}$ incubator at $37^{\circ} \mathrm{C}$.

\section{Cell transfection}

Chemically synthesized miR-591 mimic, miR-591 inhibitor or miR-negative control (NC), pcDNA-vector and pcDNA-TCF4 were obtained from Guangzhou RiboBio Co., Ltd. (Guangzhou, China). Cells were transfected using Lipofectamine 2000 (Invitrogen; Thermo Fisher Scientific, Inc.), according to the manufacturer's protocol.

\section{Quantitative reverse transcription polymerase chain reaction (QRT-PCR) analysis}

Total RNA was extracted from tissues and cell lines using TRIzol ${ }^{\circledR}$ (Invitrogen; Thermo Fisher Scientific, Inc.). The total RNA was reverse transcribed to cDNA using the Prime Script RT reagent kit (Takara Bio, Inc., Otsu, Japan) according to the manufacturer's protocol. The qRT-PCR reaction was performed using the SYBR Premix Ex Taq (Takara Bio, Inc., Otsu, Japan) on an ABI 7500 Sequence Detection system (Applied Biosystems). The U6 snRNA was used as an internal control. The thermocycling conditions was as follow: $95^{\circ} \mathrm{C}$ for $30 \mathrm{~s}$, followed by 40 cycles of $95^{\circ} \mathrm{C}$ for $5 \mathrm{~s}$ and $60^{\circ} \mathrm{C}$ for $30 \mathrm{~s}$, and one cycle of $95{ }^{\circ} \mathrm{C}$ for $15 \mathrm{~s}, 60{ }^{\circ} \mathrm{C}$ for $60 \mathrm{~s}$ and $95{ }^{\circ} \mathrm{C}$ for $15 \mathrm{~s}$ for dissociation. The relative mRNA expression was calculated using the $2^{-\Delta \Delta C q}$ methods.

\section{Cell proliferation assays}

Cell proliferation ability were assessed by CCK8 cell proliferation. Transfected cells $\left(3 \times 10^{3}\right.$ cells/per well $)$ were seeded in six-well plates. Cells were cultured for 0 , 24, 48 and $72 \mathrm{~h}$. Then, CCK8 solution $(10 \mu \mathrm{l})$ was added into each well to incubate for $2 \mathrm{~h}$ at $37^{\circ} \mathrm{C}$, the plates were detected at indicated time $(0,24,48$ and $72 \mathrm{~h})$ using a microplate reader at a wavelength of $450 \mathrm{~nm}$.

\section{Transwell invasion assay}

After cells were transfected with miR-591 mimic, miR591 inhibitor or miR-NC for $24 \mathrm{~h}$, cells were seeded in Matrigel-coated chambers $(8 \mu \mathrm{m}$ pore size, BD Bioscience, USA). $1 \times 10^{5}$ MCF-7 cells or MDA-MB-231 cells were added in the upper chamber with serum-free medium. Medium containing 10\% FBS was placed in the lower chambers to act as a chemoattractant. After $48 \mathrm{~h}$, cells were fixed and then stained with $0.1 \%$ crystal violet. Cell invasing number was assessed at five fields under a light microscope (Ti; Nikon Corporation, Tokyo, Japan) at magnification of $200 \times$.

\section{Dual luciferase reporter assay}

Potential miR-591 binding sites were predicted using TargetScan (http://www.targetscan.org). The sequence of TCF4 with the wild-type or mutant $3^{\prime}$ UTR seed region was synthesized and cloned into a pMIR-REPORT luciferase vector (Applied Biosystems; Thermo Fisher Scientific, Inc.). Then, The TCF4 with the wild-type or mutant 3'UTR pMIR-REPORT vector was co-transfected into MCF-7 cells with miR-591 mimic or miR-NC using Lipofectamine 2000 (Invitrogen; Thermo Fisher Scientific, Inc.). Firefly luciferase activity was detected at $48 \mathrm{~h}$ after 
transfection normalized against Renilla luciferase activity by using the Dual Luciferase Reporter Assay system (Promega Corporation, Madison, WI, USA) according to the manufacturer's protocol.

\section{Western blotting analysis}

Total protein was extracted from transfected cells by using a lysis buffer containing $150 \mathrm{mmol} / \mathrm{l} \mathrm{NaCl}$, $50 \mathrm{mmol} / \mathrm{l}$ Tris (pH 7.4), 1\% Triton X-100, 1\% sodium deoxycholate, $0.1 \%$ SDS, and protease inhibitor cocktail (Sigma-Aldrich; Merck KGaA, Darmstadt, Germany). Protein concentration was detected by using bicinchoninic acid assay kit (BCA) (Beyotime Institute of Biotechnology, Haimen, China). A total $30 \mu \mathrm{g}$ protein was added on $10 \%$ polyacrylamide gel and transfer to a polyvinylidene (PVDF) membrane. The membranes were blocked with 5\% skimmed milk (Sigma-Aldrich; Merck KGaA, Darmstadt, Germany) for $1 \mathrm{~h}$. Then, the membranes were incubated with primary antibody against TCF4 (Abcam, Cambridge, UK), YAP1 (Santa Cruz Biotechnology, Inc.), LATS1 (Santa Cruz Biotechnology, Inc.) and GAPDH (Cell Signaling Technology Inc.) at $4{ }^{\circ} \mathrm{C}$ overnight. Subsequently, the membranes were incubated with the corresponding horseradish peroxidase (HRP)conjugated secondary antibody (Epitomics; Abcam, Cambridge, UK) at room temperature. The membranes were detected by using an enhanced chemiluminescence detection kit (Pierce; Thermo Fisher Scientific, Inc.) and the GAPDH expression was used as an internal control. Each experiment was repeated three times.

\section{Tumorigenesis in nude mice}

All animal experiments were conducted in accordance with current Chinese regulations and standards regarding the use of laboratory animals, and all animal procedures were approved by The First Affiliated Hospital of Jinan University Animal Care and Use Committee (the protocol number IACUC-20180101-01). 4-weekold BABL/c nude mice were purchased from the Center of Laboratory Animal Science of Guangdong (Guangzhou, China). Xenograft tumors were generated by subcutaneous injection of $1 \times 10^{6}$ stable MCF-7 cells of miR-vector and miR-mimic on the hindlimbs. After 4 weeks, all mice were euthanized by dislocating the cervical spine. Tumor size was measured by a slide caliper (volume $=$ length $\times$ width $\times$ height $)$.

\section{Statistical analysis}

All results are showed as the mean \pm standard deviation (SD). Differences between groups were assessed using SPSS software (version 13.0; SPSS, Inc., Chicago, IL, USA). Data were analyzed with Student's $t$ test between two groups or one-way analysis of variance among more than two groups. Students-Newman-Keuls was performed to compare among more than two groups. $\mathrm{P}<0.05$ was considered to indicate a statistically significant difference.

\section{Results}

MiR-591 expression is downregulated in breast cancer tissues and cells

To explore the clinical role in breast cancer, we examined the miR-591 expression in breast cancer tissues and adjacent normal tissues by qRT-PCR methods. The results observed that miR-591 expression is dramatically downregulated in breast cancer than that in adjacent normal tissues (Fig. 1a). The mean level of miR-591 expression in breast cancer tissues was used as a threshold to divide patients into two groups (lower and higher expression groups). Association between miR-591 expression and clinical data of patients with breast cancer was analyzed. Results showed that miR-591 expression significantly correlated with advanced TNM stage $(\mathrm{P}=0.011)$ and lymph node metastasis $(\mathrm{P}=0.005)$ of patients (Table 1$)$.

\section{MiR-591 affects cell proliferation and invasion ability}

Furthermore, we analyzed the expression of miR-591 in four human BC cell lines MCF-7, MDA-MB-231, T-47D, SKBR3 and a human normal breast epithelial cell line MCF-10A. The qRT-PCR assay results indicated that miR-591 expression was lower in breast cancer cells compared to MCF-10A cells (Fig. 1b). To evaluate the effects of miR-591 expression on cell proliferation and invasion, we performed gain and loss function assays. The results showed that miR-591 mimic transfected MCF-7 and MDA-MB-231 cells had a dramatic higher miR-591 expression, but miR-591 inhibitor transfected MCF-7 and MDA-MB-231 cells presented a lower miR591 expression, compared to corresponding controls, respectively (Fig. 1c, d). Subsequently, the cell proliferation and invasion ability of MCF-7 and SKBR3 cells were determined using CCK8 assay and transwell assays. MCF-7 and SKBR3 cells transfected with miR-591 mimic were significantly reduced cell proliferation ability compared with the control cells at $48 \mathrm{~h}$ and $72 \mathrm{~h}$, whereas cells transfected with the miR-591 inhibitor were significantly increased cell proliferation ability compared with the control cells (Fig. 2a, b). Furthermore, transfection of miR-591 mimic in MCF-7 and SKBR3 cells significantly inhibited cell invasion ability compared with the control cells at $48 \mathrm{~h}$, whereas transfection of miR-591 inhibitor in MCF-7 and SKBR3 cells significantly enhancing cell invasion ability compared with the control cells (Fig. 2c, d). Thus, these results indicated that miR-591 inhibited cell proliferation and invasion ability of breast cancer. 

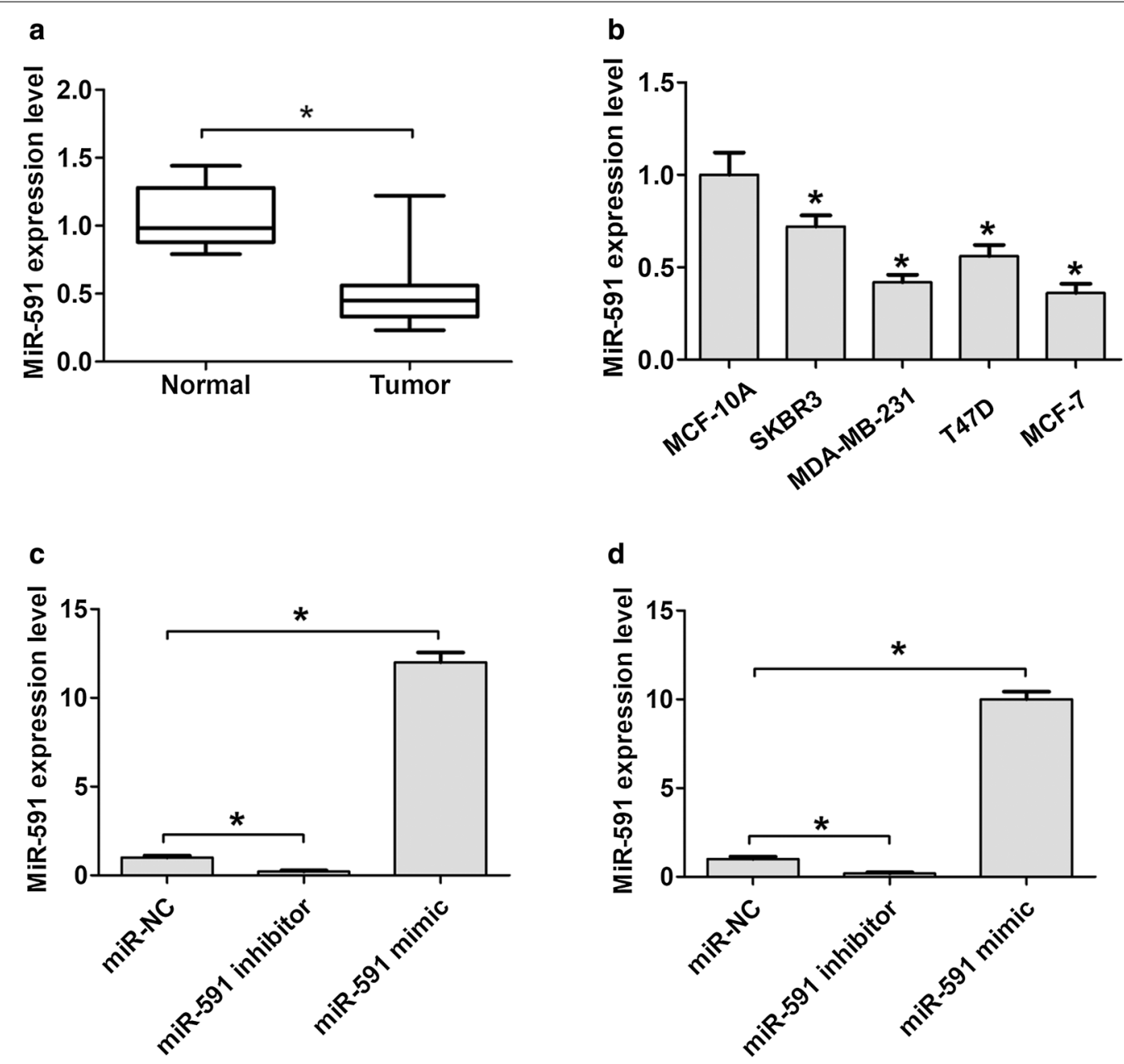

MCF-7

d

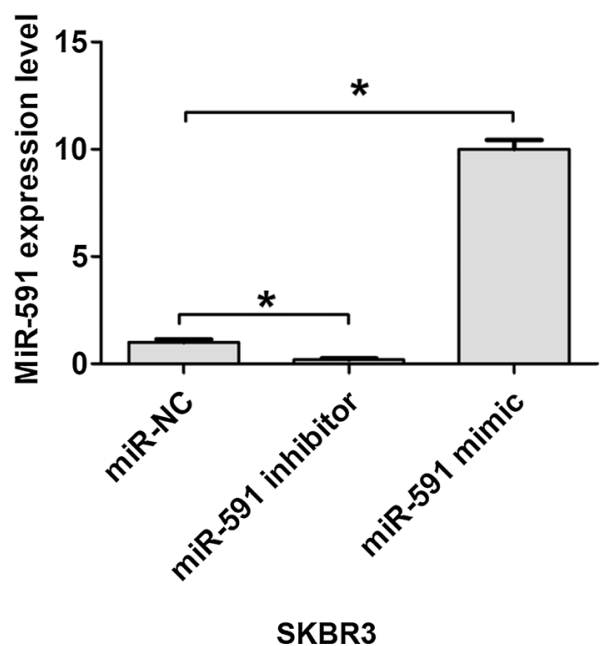

Fig. 1 Decreased miR-591 expression in breast cancer tissues and cell lines. a miR-591 expression levels in breast cancer and adjacent normal tissues was detected by qRT-PCR. b The miR-591 expression levels were detected in MCF-10A, T-47D, MDA-MB-231 or MCF7 cell lines. c The miR-591 expression levels were detected in MCF-7 cells after cells were transfected with miR-591 mimic, miR-591 inhibitor or miR-NC. d The miR-591 expression levels were detected in SKBR3 cells after cells were transfected with miR-591 mimic, miR-591 inhibitor or miR-NC. All the data are expressed as the mean $\pm S D$. ${ }^{*} P<0.05$. qRT-PCR quantitative real-time $P C R, S D$ standard deviation

\section{Upregulation of miR-591 suppresses the proliferation of breast cancer cells in vivo}

Furthermore, xenograft tumors were generated by subcutaneous injection of $1 \times 10^{6}$ stable MCF-7 cells of miR-vector and miR-mimic on the hindlimbs. After 4 weeks, all mice were euthanized by dislocating the cervical spine. Tumor size was measured by a slide caliper (volume $=$ length $\times$ width $\times$ height). Our results demonstrated that miR-591 mimic group showed much smaller tumors volume than that in miR-NC group (Fig. 3a). In addition, the tumor volume grown slower in miR-591 mimic group compared to that in miRNC group (Fig. 3b). Thus, these results indicated that upregulation of miR-591 suppressed the proliferation of breast cancer cells in vivo.

\section{TCF4 is a direct target of miR-591 in breast cells}

By online databases (TargetScan and miRDB) predicted relative target of miR-591, we found that Transcription factor 4 (TCF4) was identified as a potential target of miR-591 (Fig. 4a). Furthermore, we co-transfected of TCF4-WT and miR-591 mimic in MCF-7 cells resulted in lower luciferase activity, while co-transfected of TCF4MUT and miR-591 mimic showed no change in luciferase activity (Fig. 4b). Moreover, we detected the mRNA expression of TCF4 after upregulation of miR-591 or 
Table 1 The association of clinicopathological factors with miR-591 expression in 78 breast cancer patients

\begin{tabular}{|c|c|c|c|c|}
\hline \multirow[t]{2}{*}{ Characteristics } & \multirow[t]{2}{*}{ Total } & \multicolumn{2}{|c|}{ MiR-591 expression level } & \multirow[t]{2}{*}{ P-value } \\
\hline & & Lower $(n=38)$ & Higher $(n=40)$ & \\
\hline \multicolumn{4}{|l|}{ Age (years) } & 0.267 \\
\hline$\leq 45$ & 32 & 18 & 14 & \\
\hline$>45$ & 46 & 20 & 26 & \\
\hline \multicolumn{4}{|l|}{ Tumor size $(\mathrm{cm})$} & 0.519 \\
\hline$<2$ & 30 & 16 & 14 & \\
\hline$>2$ & 48 & 22 & 26 & \\
\hline \multicolumn{4}{|l|}{ PR status } & 0.223 \\
\hline No & 30 & 12 & 18 & \\
\hline Yes & 48 & 26 & 22 & \\
\hline \multicolumn{4}{|l|}{ TNM stage } & $0.011^{*}$ \\
\hline |/II & 50 & 19 & 31 & \\
\hline III & 28 & 19 & 9 & \\
\hline \multicolumn{4}{|c|}{ Lymph node metastasis } & $0.005^{*}$ \\
\hline No & 53 & 20 & 33 & \\
\hline Yes & 25 & 18 & 7 & \\
\hline \multicolumn{4}{|l|}{ Bone metastasis } & 0.615 \\
\hline No & 70 & 34 & 36 & \\
\hline Yes & 8 & 4 & 4 & \\
\hline \multicolumn{4}{|l|}{ ER } & 0.972 \\
\hline No & 45 & 22 & 23 & \\
\hline Yes & 33 & 16 & 17 & \\
\hline \multicolumn{4}{|c|}{ Histological grade } & 0.262 \\
\hline $\mathrm{G} 1 / 2$ & 52 & 23 & 29 & \\
\hline G3 & 26 & 15 & 11 & \\
\hline
\end{tabular}

$E R$ estrogen receptor, $P R$ progesterone receptor * $\mathrm{P}<0.05$

downregulation of miR-591 in MCF-7 or SKBR3 cells by qRT-PCR analysis. We found that transfection of miR-591 mimic in MCF-7 or SKBR3 cells significantly downregulated the mRNA expression of TCF4 (Fig. 4c, d). Transfection of MiR-591 inhibitor in MCF-7 cells resulted in upregulation of TCF4 expression (Fig. 4c, d). In additional, we also demonstrated that transfection of miR-591 mimic significantly downregulated the protein expression of TCF4 in MCF-7 or SKBR3 cells compared to the control groups (Fig. 4e, f). Thus, these results indicated that TCF4 was a direct target of miR-591 in breast cells.

TCF4 up-regulation partially relieves miR-591 mediated suppression of cell proliferation and invasion in breast cancer

To determine whether miR-591 suppresses cancer cell proliferation and invasion by regulating TCF4. We constructed TCF4 over-expressing vector and transfected into MCF-7 cells. Over-expression of TCF4 was confirmed by qRT-PCR analysis (Fig. 5a). The CCK8 cell proliferation and transwell assays showed that miR591-mediated suppression of cell proliferation and invasion was partially rescued by overexpressed TCF4 in MCF-7 cells (Fig. 5b, d). Together, these data indicate that miR-591 suppresses cell proliferation and invasion by down-regulating TCF4.

\section{MiR-591 inhibits Hippo-YAP signaling pathway in breast cancer}

Hippo-YAP signaling pathway has been reported to involved in tumor progression in breast cancer [13]. We detected the YAP expression by upregulation of miR-591 in MCF-7 and SKBR3 cells. The western blot analysis showed that YAP1 expression was downregulated after miR-591 upregulation in MCF-7 or SKBR3 cells compared to the control groups (Fig. 6a, b). However, LAST1 expression was upregulated after miR-591 upregulation in MCF-7 or SKBR3 cells compared to the control groups (Fig. 6a, b). Thus, these results indicated that miR-591 inhibited Hippo-YAP signaling pathway in breast cancer.

\section{Discussion}

Breast cancer is a heterogeneous disease that includes various subtypes, with different biological behavior and clinical outcome. miRNAs have been found to be participated in breast cancer progression. MiR-591 have important roles in several tumors such, as miR-591 confers PTX resistance to ovarian cancer cells and modulation of MiR-591 resensitises PTX-resistant cancer cells by targeting ZEB1 [11]. However, the clinical role and functional effects of miR-591 in breast cancer remains unknown.

In the present study, we have ascertained novel molecular mechanisms miR-591 inhibited cell proliferation and invasion in breast cancer. We found that miR-519 expression was low in breast cancer tissues and cells. Lower miR-591 expression notably related to lymph node metastasis and TNM stage. Bone metastasis is frequent in breast cancer patients, in the study, the clinical analysis results showed no association between miR-519 expression and bone metastasis. We speculated the cases number may be inadequate and needed to extend the cases in the further. Moreover, the cell lines use in the study is not association with osteotropism, we did evaluate the expression of miR-591 is lower in several breast cancer cell lines. In vitro, cell proliferation and invasion were inhibited by transfection of miR-591 mimic in breast cancer. In vivo, our results also indicated that upregulation of miR-591 suppressed the proliferation of breast cancer cells in vivo. Moreover, we identified that TCF4 was a target of miR-591 in breast cancer. TCF4 play crucial functional role in breast cancer. The TCF4/ $\beta$-catenin pathway 


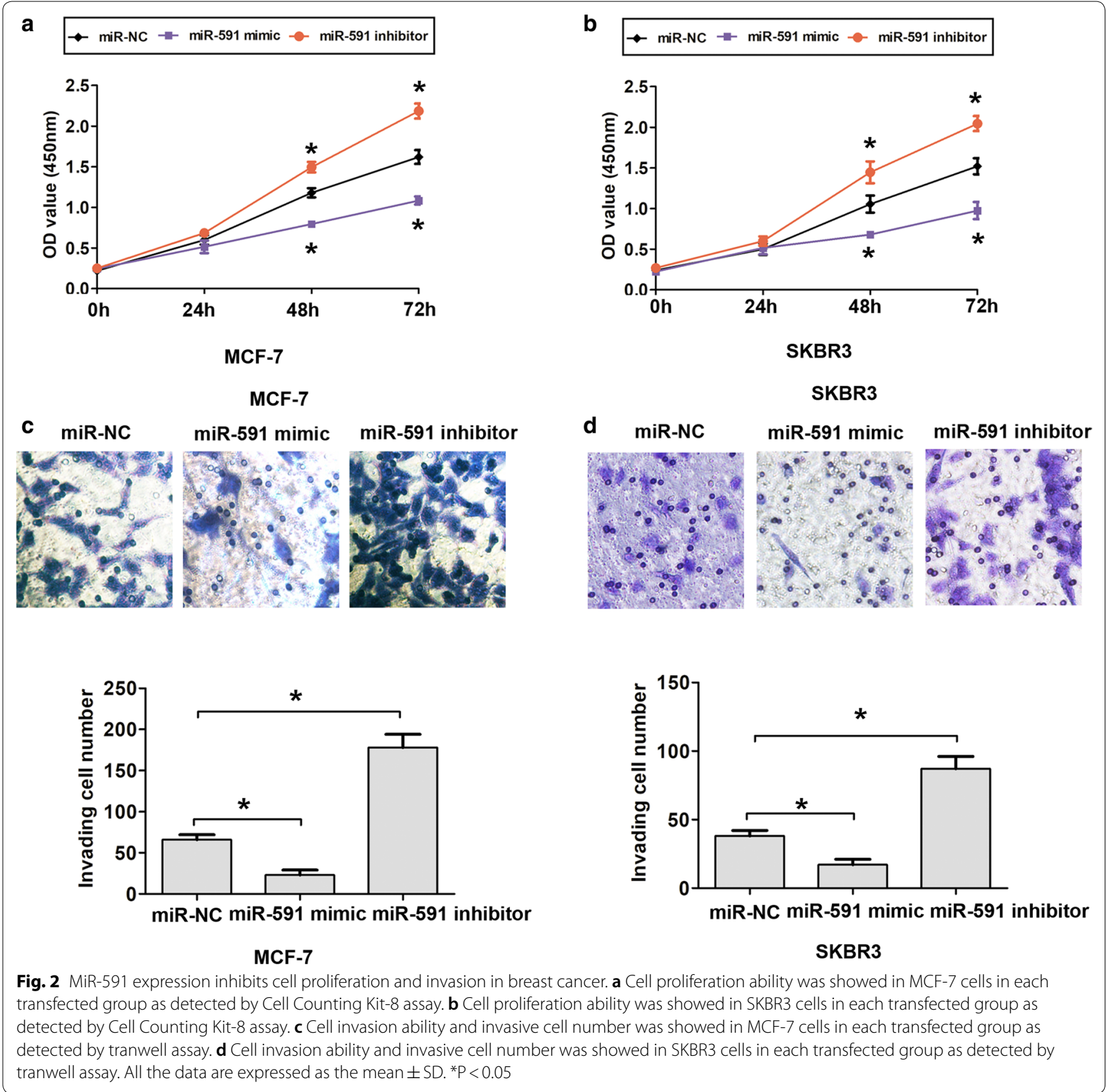

and chromatin structure cooperate to regulate D-glucuronyl C5-epimerase expression in breast cancer [14]. MicroRNA-100 suppresses the migration and invasion of breast cancer cells by targeting FZD-8 and inhibiting T-cell factor-4 (TCF-4) expression [15]. By upregulating the TCF4 expression, we demonstrated that TCF4 mediated the inhibiting effects of miR-591 expression on cell proliferation and invasion in breast cancer cells.

Besides, we demonstrated that miR-591 overexpression inhibited the Hippo-YAP/TAZ signaling pathway in breast cells by downregulated YAP1 expression. Studies found that the Hippo-YAP/TAZ pathway mediates geranylgeranylation signaling in breast cancer progression [16]. Notch3 inhibits epithelial-mesenchymal transition by activating Kibra-mediated Hippo/YAP signaling in breast cancer epithelial cells [13]. Dual inhibition of WNT and Yes-associated protein signaling retards the growth of triple-negative breast cancer in both mesenchymal and epithelial states [17]. Thus, these results indicated that upregulation of miR-591 could inhibit the TCF4 and Hippo-YAP signaling, which may function as a potential target of breast cancer treatment. 
a

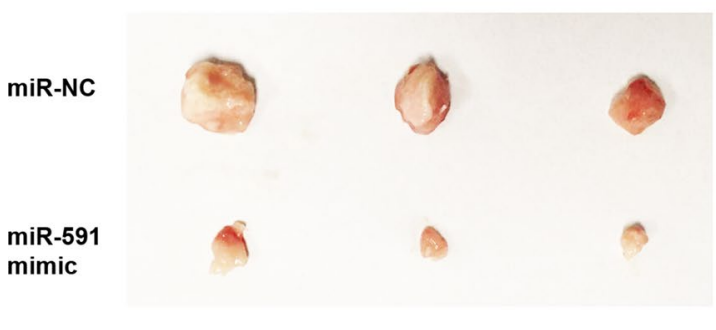

b $\quad \rightarrow$ miR-NC

$\rightarrow$ miR-591 mimic

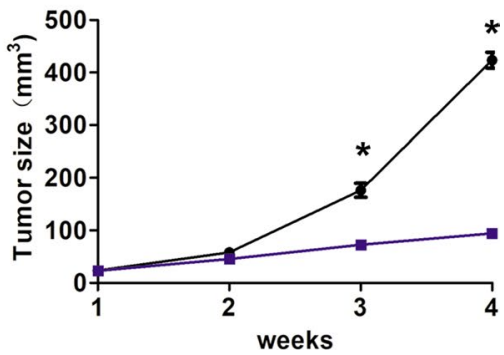

Fig. 3 Upregulation of miR-591 suppresses the proliferation of breast cancer cells in vivo. a MiR-591 mimic group showed much smaller tumors volume than that in miR-NC group. b The tumor volume grown slower in miR-591 mimic group compared to that in miR-NC group. ${ }^{*}<<0.05$

a

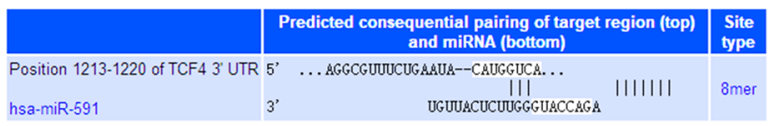

C

MCF-7

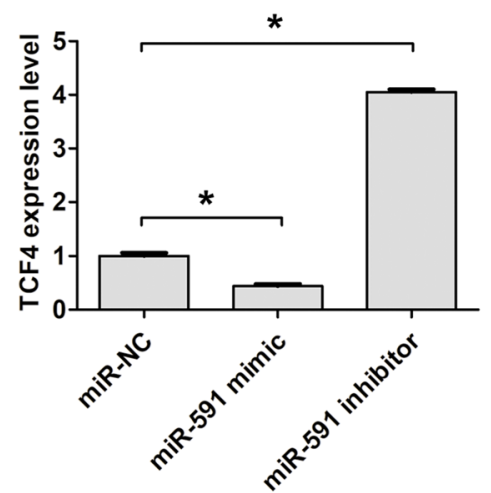

e

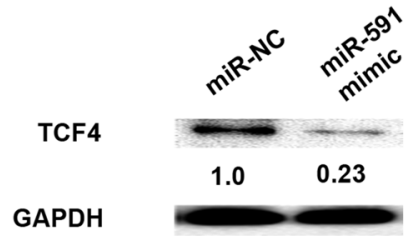

b

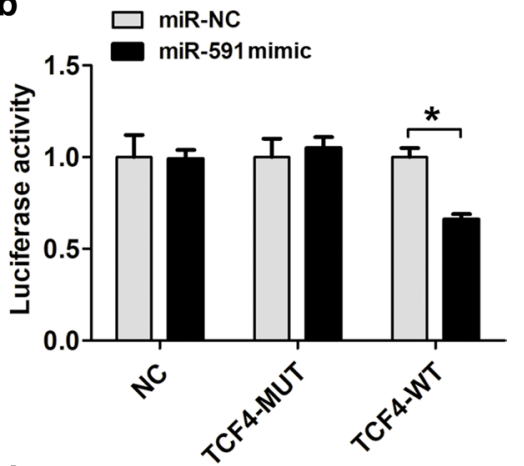

d

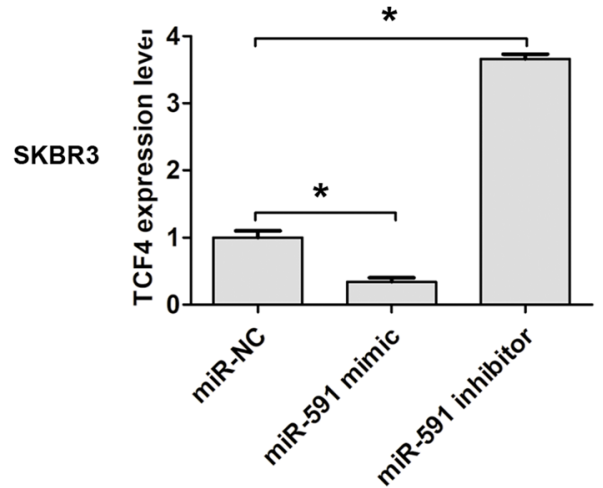

f

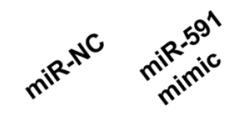

TCF4

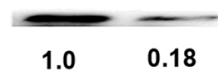

GAPDH

SKBR3

Fig. 4 TCF4 is as target of miR-591 in breast cancer. a The miR-591-binding sites in the 3'-UTR of TCF4 was shown. b MCF-7 cells were co-transfected with pMIR-REPORT-TCF4-3'UTRWT or PMIR-REPORT-TCF4-3'UTR MUT, together with miR-591 mimic or miR-NC. Luciferase activity was measured at $48 \mathrm{~h}$ after transfection. c, d TCF4 mRNA expression in MCF-7 or SKBR3 cells transfected with miR-591 mimic, miR-591 inhibitor or miR-NC was measured using qRT-PCR. e, fTCF4 protein expression in MCF-7 or SKBR3 cells transfected with miR-591 mimic or miR-NC was measured using western blot analysis. All the data are expressed as the mean $\pm \mathrm{SD}$. ${ }^{*} \mathrm{P}<0.05$ 
a

a

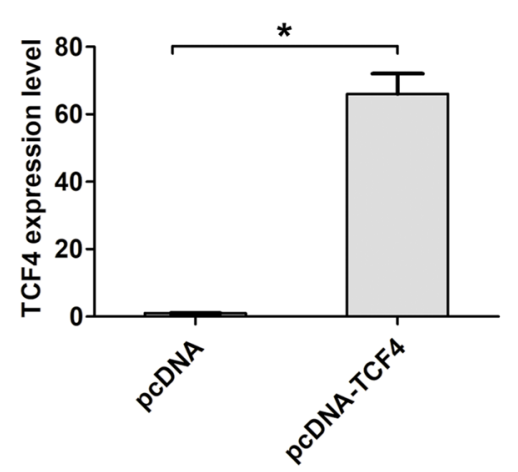

C

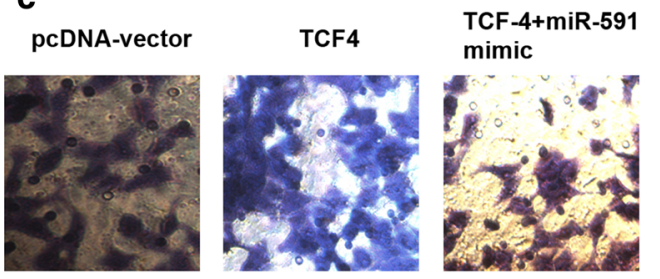

MCF-7 b

$\rightarrow$ pcDNA $\rightarrow$ pcDNA-TCF4 $₫$ TCF4+miR-591 mimic

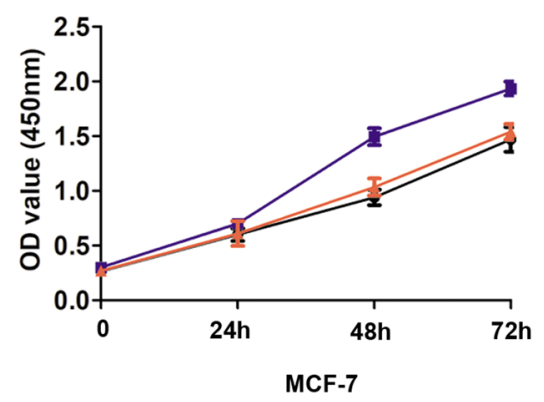

d

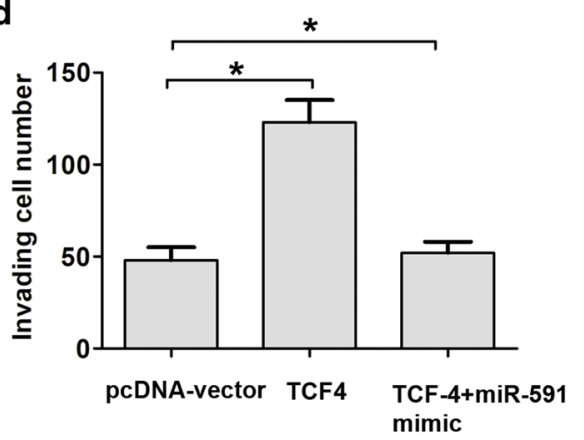

MCF-7

Fig. 5 TCF4 up-regulation partially relieves miR-591 mediated suppression of cell proliferation and invasion in breast cancer. a TCF4 mRNA expression in MCF-7 cells transfected with pCDNA-vector or pCDNA-TCF4 was measured using qRT-PCR. $\mathbf{b}$ Cell proliferation ability was showed in MCF-7 cells in transfected group including pcDNA-vector, pcDNA-TCF4, TCF4 + miR-591 mimic as detected by Cell Counting Kit-8 assay. $\mathbf{c}$, $\mathbf{d}$ Cell invasion ability was showed in MCF-7 cells in transfected group including pcDNA-vector, pcDNA-TCF4, TCF4+ miR-591 mimic as detected by transwell assay. All the data are expressed as the mean $\pm \mathrm{SD}$. ${ }^{*} \mathrm{P}<0.05$

a

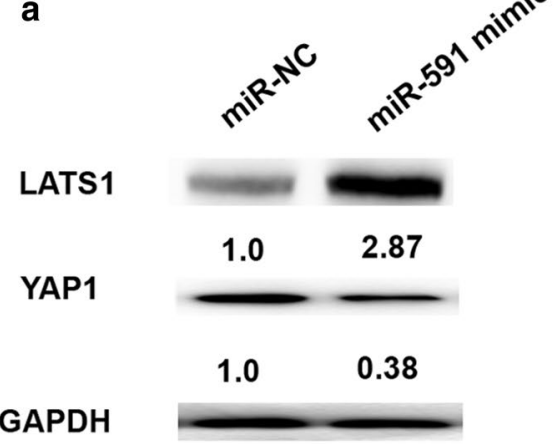

MCF-7 b

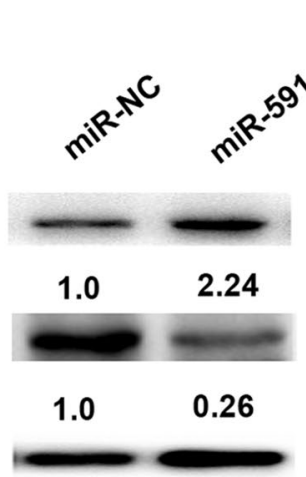

SKBR3

Fig. 6 MiR-591 inhibits Hippo-YAP signaling pathway in breast cancer. a LATS1 and YAP1 protein expression in MCF-7 cells transfected with miR-591 mimic or miR-NC was measured using western blot analysis. b LATS1 and YAP1 protein expression in SKBR3 cells transfected with miR-591 mimic or miR-NC was measured using western blot analysis 


\section{Conclusion}

In conclusion, our results indicated that miR-591 expression was downregulated in breast cancer tissues and cells. Furthermore, we demonstrated that miR-591 could regulate cell proliferation and invasion by targeting TCF4 and inhibited the TCF4 and Hippo-YAP signaling, which may function as a potential target of breast cancer treatment.

\section{Abbreviations \\ MiRNAs: microRNA; DMEM: Dulbecco's modified Eagle's medium; FBS: fetal bovine serum; TCF4: transcription factor 4.}

\section{Authors' contributions}

$\mathrm{XH}$ and FT designed the experiments; ZW, MZ, and QZ conducted experiments; $\mathrm{XH}$ and FT provided research materials and methods; $\mathrm{XH}$ and $\mathrm{FT}$ analyzed data; $\mathrm{XH}$ and FT wrote the manuscript. All authors read and approved the final manuscript.

\section{Author details}

${ }^{1}$ Department of Breast Surgery, The First Affiliated Hospital of Jinan University, 613 West Huangpu Road, Guangzhou 510630, Guangdong, People's Republic of China. ${ }^{2}$ Department of Pathology, The First Affiliated Hospital of Jinan University, Guangzhou 510630, Guangdong, People's Republic of China. ${ }^{3}$ Department of Experimental Center, The First Affiliated Hospital of Jinan University, Guangzhou 510630, Guangdong, People's Republic of China.

\section{Acknowledgements}

Not applicable.

\section{Competing interests}

The authors declare that they have no competing interests.

\section{Availability of data and materials}

We declared that materials described in the manuscript, including all relevant raw data, will be freely available to any scientist wishing to use them for noncommercial purposes, without breaching participant confidentiality.

\section{Consent for publication}

Not applicable.

\section{Ethics approval and consent to participate}

The study was approved by the Ethical Committee of The First Affiliated Hospital of Jinan University and all of the patients provided written informed consent.

\section{Funding}

Not applicable.

\section{Publisher's Note}

Springer Nature remains neutral with regard to jurisdictional claims in published maps and institutional affiliations.

Received: 19 October 2018 Accepted: 8 April 2019

Published online: 24 April 2019
References

1. Anastasiadi Z, Lianos GD, Ignatiadou E, Harissis HV, Mitsis M. Breast cancer in young women: an overview. Updat Surg. 2017;69(3):313-7.

2. Ferlay J, Soerjomataram I, Dikshit R, Eser S, Mathers C, Rebelo M, Parkin DM, Forman D, Bray F. Cancer incidence and mortality worldwide: sources, methods and major patterns in GLOBOCAN 2012. Int J Cancer. 2015:136(5):E359-86.

3. Marquette C, Nabell L. Chemotherapy-resistant metastatic breast cancer. Curr Treat Options Oncol. 2012;13(2):263-75.

4. Dong XY, Guo P, Boyd J, Sun X, Li Q, Zhou W, Dong JT. Implication of snoRNA U50 in human breast cancer. J Genet Genom. 2009;36(8):447-54.

5. Yates LA, Norbury CJ, Gilbert RJ. The long and short of microRNA. Cell. 2013;153(3):516-9.

6. Di Leva G, Garofalo M, Croce CM. MicroRNAs in cancer. Ann Rev Pathol. 2014;9:287-314.

7. Esquela-Kerscher A, Slack FJ. Oncomirs-microRNAs with a role in cancer. Nat Rev Cancer. 2006;6(4):259-69.

8. Li N, Wang L, Tan G, Guo Z, Liu L, Yang M, He J. MicroRNA-218 inhibits proliferation and invasion in ovarian cancer by targeting Runx2. Oncotarget. 2017:8(53):91530-41.

9. Pajic M, Froio D, Daly S, Doculara L, Millar E, Graham PH, Drury A, Steinmann $A$, de Bock CE, Boulghourjian A, et al. miR-139-5p modulates radiotherapy resistance in breast cancer by repressing multiple gene networks of DNA repair and ROS defense. Can Res. 2017;78(2):501-15.

10. Li J, Lai Y, Ma J, Liu Y, Bi J, Zhang L, Chen L, Yao C, Lv W, Chang G, et al. miR-17-5p suppresses cell proliferation and invasion by targeting ETV1 in triple-negative breast cancer. BMC cancer. 2017:17(1):745.

11. Huh JH, Kim TH, Kim K, Song JA, Jung YJ, Jeong JY, Lee MJ, Kim YK, Lee $\mathrm{DH}, \mathrm{An} \mathrm{HJ}$. Dysregulation of miR-106a and miR-591 confers paclitaxel resistance to ovarian cancer. Br J Cancer. 2013;109(2):452-61.

12. Cheng $S, X u Y$, Shi $Z$, Lin $Y$, Hoang $C D$, Zhang $X$. Overexpression of micro ribonucleic acid-591 inhibits cell proliferation and invasion of malignant pleural mesothelioma cells. Thoracic Cancer. 2016;7(3):340-7.

13. Zhang X, Liu X, Luo J, Xiao W, Ye X, Chen M, Li Y, Zhang GJ. Notch3 inhibits epithelial-mesenchymal transition by activating Kibra-mediated Hippo/YAP signaling in breast cancer epithelial cells. Oncogenesis. 2016;5(11):e269.

14. Mostovich LA, Prudnikova TY, Kondratov AG, Gubanova NV, Kharchenko OA, Kutsenko OS, Vavilov PV, Haraldson K, Kashuba VI, Ernberg I, Zabarovsky ER. The TCF4/ $\beta$-catenin pathway and chromatin structure cooperate to regulate $\mathrm{D}$-glucuronyl $\mathrm{C} 5$-epimerase expression in breast cancer. Epigenetics. 2012;7(8):930-9.

15. Jiang $Q$, He $M$, Guan $S, M a ~ M, W u ~ H, Y u Z$, Jiang $L$, Wang $Y$, Zong $X$, Jin F, Wei M. MicroRNA-100 suppresses the migration and invasion of breast cancer cells by targeting FZD-8 and inhibiting Wnt/ $\beta$-catenin signaling pathway. Tumor Biol. 2016;37(4):5001-11.

16. Lin $Q$, Yang W. The Hippo-YAP/TAZ pathway mediates geranylgeranylation signaling in breast cancer progression. Mol Cell Oncol. 2016;3(3):e969638

17. Sulaiman A, McGarry S, Li L, Jia D, Ooi S, Addison C, Dimitroulakos J, Arnaout A, Nessim C, Yao Z, et al. Dual inhibition of Wnt and Yes-associated protein signaling retards the growth of triple-negative breast cancer in both mesenchymal and epithelial states. Mol Oncol. 2018;12(4):423-40.

Ready to submit your research? Choose BMC and benefit from:

- fast, convenient online submission

- thorough peer review by experienced researchers in your field

- rapid publication on acceptance

- support for research data, including large and complex data types

- gold Open Access which fosters wider collaboration and increased citations

- maximum visibility for your research: over 100M website views per year

At BMC, research is always in progress.

Learn more biomedcentral.com/submissions 